\title{
L'enzymogramme de la phosphatase alcaline du lait de vache et sa modification pendant le métissage
}

\author{
par \\ L.M. BURUIANA et Marguerite MARIN \\ Laboratoire de Biochimie, Faculté de Médecine Vétérinaire \\ de Bucarest, R.S. Roumanie
}

La biosynthèse des enzymes comme celle de toute matière protéique est réglée génétiquement. Le polymorphisme de certains enzymes pourrait donc nous informer sur la structure ou arrangement du ADN chromosomial. Parce que les caractères morphophysiologiques du bétail dépendent du type de l'ADN héréditaire, on comprend pourquoi la recherche du polymorphisme enzymatique et sa modification à la suite des influences du milieu extérieur pourrait présenter une certaine importance dans les opérations d'amélioration des races d'animaux. La mise en évidence des formes moléculaires multiples des enzymes, les isoenzymes, est de plus en plus employée pour dépister et suivre les troubles et les modifications métaboliques, et on connaît beaucoup de travaux qui établissent des corrélations entre ces diverses modifications et le spectre des isoenzymes ou l'enzymogramme respectif. Des recherches antérieures $[3,4]$ nous ont prouvé que, pendant le métissage de certaines races d'animaux domestiques, il se produit une modification spécifique de l'enzymogramme de la cathépsine tissulaire.

Dans le présent travail, nous exposons les résultats obtenus quant à la modification de l'enzymogramme de la phosphatase alcaline du lait de vache chez quelques races pures et leur métis.

Quoique le polymorphisme de la phosphatase alcaline et acide ait fait l'objet de recherches dans le cas des tissus et du sang $(5,8,11)$, il n'existe pas dans le cas du lait, d'après nos connaissances, de recherches similaires.

A l'exception des laits de jument et d'ânesse [1], le lait d'autres espèces est riche en phosphatase, sa présence étant étroitement liée avec les réactions métaboliques de la glande mammaire. 
Son activité est très variable et jusqu'à aujourd'hui, on n'a trouvé aucune corrélation pertinente entre son activité et certains indices morpho-physiologiques ou avec les troubles pathologiques de la glande mammaire. En ce qui concerne le $p H$ optimum nous avons trouvé [1] un seul maximum $(p H$ 9,25) pour le lait de vache en opposition avec les laits de chèvre, truie et femme qui présentent 2 maxima, un dans la zone acide $(p \mathrm{H} 5)$ et l'autre dans la zone alcaline ( $p H 8,92)$ (voir fig. 1).

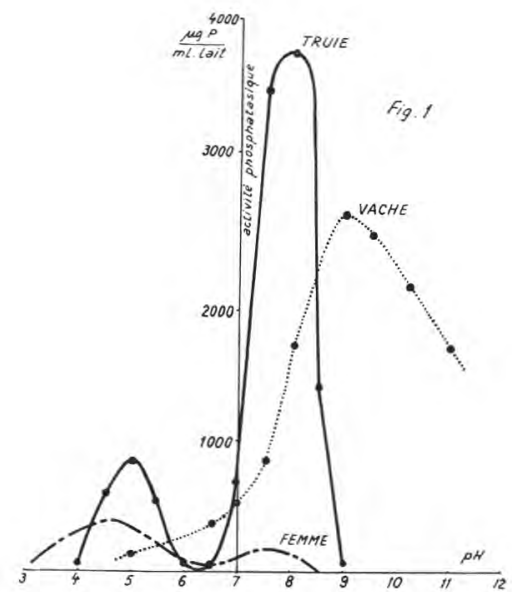

Fig. 1. - Variation de l'activité de la phosphatase du lait avec le $\mathrm{pH}$ chez diverses espèces.

Certains chercheurs ont réussi à isoler du colostrum une phosphatase acide analogue à celle de la prostate, avec le maximum d'activité à $p H$ 4,5 étant inhibée comme celle-ci par le tartrate de soude $(6,12)$.

A l'occasion de recherches sur la phosphatase alcaline du lait [1] nous avons mis en évidence certaines de ses particularités, dont la plus intéressante est qu'elle n'a pas besoin d'être activée par les ions $\mathrm{Mg}$. Cette propriété est rencontrée seulement dans le cas des phosphatases acides (érythrocytes, prostate, sperme). Nos tentatives de séparation et purification ont démontré que la phosphatase de lait de vache est associée avec a-lactalbumine. Elle est aussi fortement absorbée par la membrane des globules gras, ce qui la protège contre la dénaturation et l'inactivation thermique. C'est pour cela que l'inactivation complète de la phosphatase de la crème nécessite une température d'environ $90^{\circ} \mathrm{C}$ [2].

\section{MATERIEL ET METHODES DE TRAVAIL}

Dans ce qui suit, nous rapportons la technique employée pour le dosage de l'activité de la phosphatase du lait de vache et celle 
de la chromatographie sur colonne. Cette dernière nous a servi pour mettre en évidence le polymorphisme de la phosphatase et les modifications qui apparaissent à la suite du métissage.

Le lait que nous avons analysé provenait des races de vaches les plus répandues actuellement en Roumanie et leurs métis.

Il s'agit des races: Brune (acclimatement de Schwyz), Tachetée de Roumanie (acclimatement de Simmental), Rouge danoise, Holstein (provenant du Canada) et des métis suivants: Brune Holstein, Tachetée - Holstein, Rouge danoise - Holstein et Brune Tachetée.

Le lait provenant des races et métis précités, fraîchement récolté à diverses périodes de lactation et pendant différentes saisons de l'année, était analysé.

Immédiatement ou après 1 - 2 heures de conservation à la glacière, on déterminait l'activité phosphatasique du lait entier et dégraissé par centrifugation pendant 30 minutes à 3000 tours/ minute. En général, le lait écrémé montrait une activité d'environ 50 p. 100 moindre, en fonction de son pourcentage en matière grasse.

L'activité phosphatasique était déterminée d'après le phénol mis en liberté du mono-phenyl-phosphate disodique. La technique employée était calquée sur celle de E.J. King \& A.R. Armstrong [9]. Nous exposons ci-après les détails.

\section{Détermination de l'activité phosphatasique.}

\section{Réactifs}

1. - Solution tampon de monophenylphosphate disodique ( $1 \mathrm{~g}$ de monophenylphosphate disodique et $10,3 \mathrm{~g}$ de véronal sodique pour 1 litre d'eau distillée). Se conserve bien à la glacière en présence du chloroforme.

2. - Réactif de Folin préparé selon les indications de l'Auteur [6].

3. - Réactif de Folin dilué 1/2, avec de l'eau distillée.

4. - Solution de carbonate de soude pur, cristallisé, à 5 p. 100 . Technique.

Placer dans un tube à essai $2 \mathrm{ml}$ de solution de monophénylphosphate disodique tamponnée (solution substrat) et chauffer à $37^{\circ}$.

Ajouter $0,2 \mathrm{ml}$ de lait et maintenir $1 / 4$ heure à la même température. Ajouter ensuite $2 \mathrm{ml}$ du réactif de Folin dilué. Agiter et filtrer.

On prélève $2,1 \mathrm{ml}$ du filtrat correspondant à $0,1 \mathrm{ml}$ de lait et on ajoute $4 \mathrm{ml}$ de la solution de carbonate de soude à 5 p. 100. On maintient 10 minutes à $50^{\circ}$ et, après refroidissement, on lit l'extinction 
au photocolorimètre dans le rouge ( $\mathrm{nm}$ 660) en opposition avec un témoin obtenu dans les mêmes conditions, avec la différence qu'on ajoutait le réactif de Folin dilué immédiatement après le mélange de substrat avec le lait. On calcule la quantité de phénol mis en liberté à la suite de l'action enzymatique de la phosphatase alcaline contenue dans le lait à l'aide d'une courbe étalon établie avec une solution de phénol de concentration connue (10 mg p. 100). On rapporte la quantité de phénol en $\mu \mathrm{g}$ pour $1 \mathrm{mg}$ de protéine. Celle-ci était déterminée par la technique de G.L. Miller [10] en utilisant comme solution étalon l'albumine cristallisée bovine. Cette technique est très indiquée dans notre cas car on utilise presque les mêmes réactifs que pour le dosage de l'activité phosphatasique.

Chromatographie de la phosphatase alcaline. Séparation des isozymes. Enzymogramme et protéinogramme.

Nous avons recouru à la méthode chromatographique par gel-filtration sur Sephadex G-200 (Pharmacia Uppsala). La colonne pour chromatographie avait les dimensions $2 \times 20 \mathrm{~cm}$. Elle était équilibrée après remplissage avec une solution tampon saline de Tris ( $\mathrm{HCl} 0,05 \mathrm{M}+\mathrm{NaCl} 0,1 \mathrm{M}$ de $p \mathrm{H} \mathrm{8,2).} \mathrm{On} \mathrm{filtrait} 10 \mathrm{ml} \mathrm{du}$ lait, dégraissé par centrifugation, et on éluait ensuite avec la solution saline tamponnée en récoltant des fractions de $3 \mathrm{ml}$. Le nombre des fractions représentant le volume nécessaire d'élution oscillait entre 15 et 19 . On déterminait dans chaque fraction l'activité phosphatasique et le contenu en matière protéique.

On traçait ensuite les courbes représentant la variation de l'activité phosphatasique (enzymogramme) en fonction du volume élué.

Les maxima de l'activité ou les pics de l'enzymogramme indiquent le nombre de fractions ou les isoenzymes de la phosphatase et, leur position, le poids moléculaire des fractions protéiques douées d'activité phosphatasique, si, bien entendu, la colonne est étalonnée au préalable avec une protéine ou plusieurs, de poids moléculaire connu. Dans quelques cas, le lait était dialysé avant d'être chromatographié. Les résultats sont différents; l'explication de ce phénomène fera l'objet d'une étude séparée. L'enzyme adsorbé par les globules gras peut être détaché par barattage. L'analyse chromatographique du petit lait ainsi obtenu donne un enzymogramme presque identique, ce qui prouve l'identité de l'enzyme adsorbé par les globules gras avec celle du lait dégraissé. La répartition est déterminée seulement par les forces interfaciales, fait d'ailleurs vérifié par la variation de l'activité phosphatasique du lait dégraissé avec la quantité et les qualités physiques des globules gras. 


\section{RESULTATS OBTENUS}

Dans les figures 2, 3, 4 et 5 sont représentés les enzymogrammes de la phosphatase du lait récolté des vaches de race pure mentionnés plus haut.
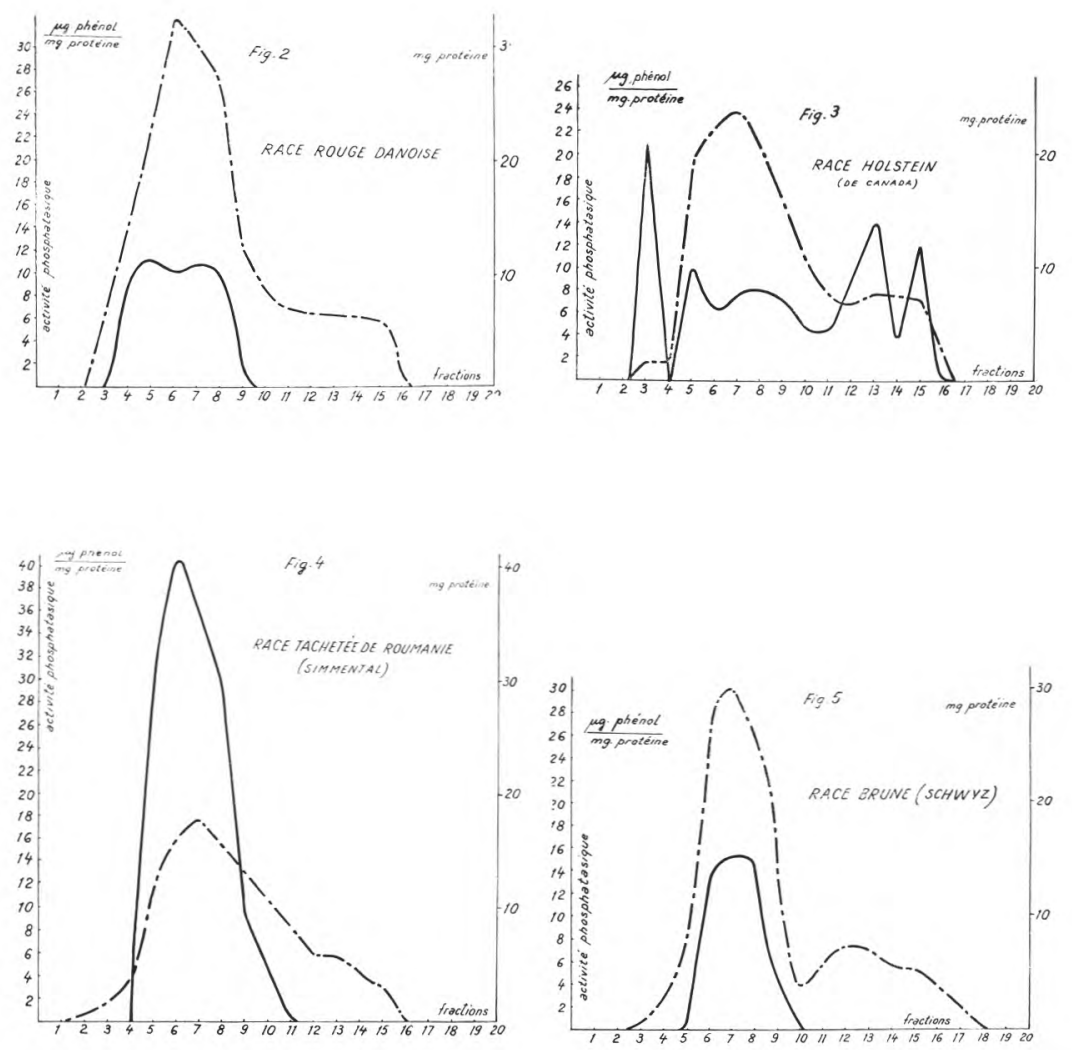

Fig. 2. - L'enzymogramme

-) de la phosphatase et le protéinogramme (-. - ) du lait de vache, race Rouge danoise.

Fig. 3. - L'enzymogramme $(\longrightarrow$ de la phosphatase et le protéinogramme (-. - ) du lait de vache, race Holstein.

Fig. 4. - L'enzymogramme (- $(-$ de la phosphatase et le protéinogramme (-.-) du lait de vache, race Tachetée de Roumanie.

Fig. 5. - L'enzymogramme $(-)$ de la phosphatase et le protéinogramme (-. - ) du lait de vache, race Brune. 
Le tracé des protéinogrammes respectifs permet de localiser l'activité enzymatique dans l'ensemble des fractions protéiques du lait et d'obtenir des indications quant à leur poids moléculaire. On remarque le polymorphisme accentué de la phosphatase du lait provenant des vaches de race Holstein (fig. 3), en opposition avec les autres races. L'effet de métissage se traduit par la modification caractéristique de l'enzymogramme des races pures participant au métissage (fig. 6, 7, 8 et 9).
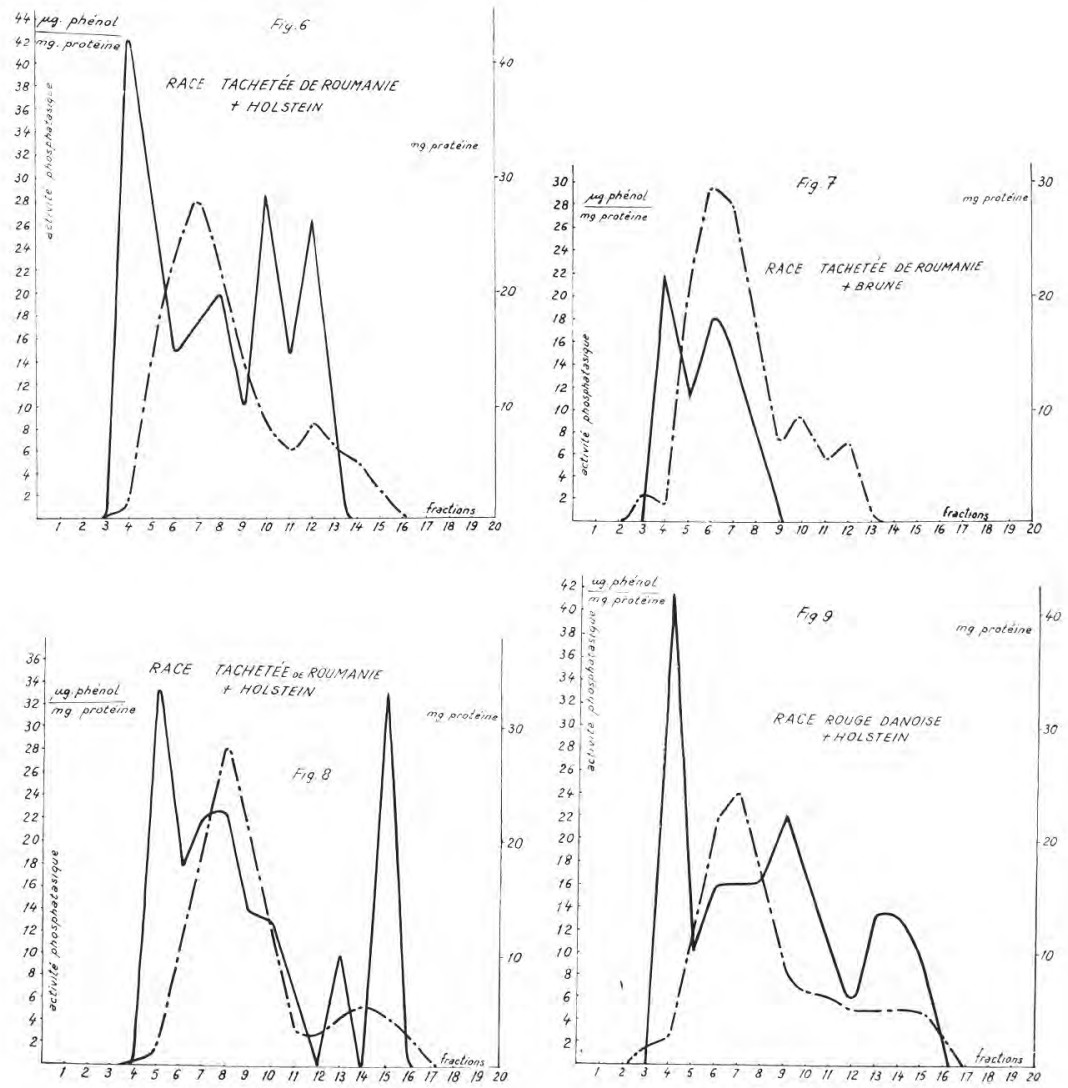

F'ig. 6. - L'enzymogramme ( ) de la phosphatase et le protéinogramme (-.-) du lait de vache, métis « Tachetée de Roumanie - Holstein».

Fig. 7. - L'enzymogramme (- $(-)$ de la phosphatase et le protéinogramme (-. - ) du lait de vache, métis «Tachetée de Roumanie - Brune».

Fig. 8. - L'enzymogramme (- ) de la phosphatase et le protéinogramme (- - - du lait de vache, métis «Holstein - Tachetée de Roumanie».

Fig. 9. - L'enzymogramme (- - ) de la phosphatase et le protéinogramme (-.-) du lait de vache, métis «Rouge danoise - Holstein». 
Les résultats sont bien caractéristiques et confirment encore une fois nos constatations antérieures $(1,2)$ quant à l'influence de l'hybridation sur la qualité de certains enzymes.

\section{DISCUSSION}

L'existence de formes moléculaires multiples des enzymes peut être expliquée soit par l'influence des facteurs d'ordre génétique (polymorphisme génétique, polyallélisme), soit comme le résultat de certaines réactions qui, en modifiant la conformation de la molécule protéique (allostérisme), change sa réactivité chimique (polymorphisme chimique, de nature métabolique).

Dans le premier cas, la matrice héréditaire, c'est-à-dire l'ADN chromosomial contient lui-même l'information nécessaire à la biosynthèse des isoenzymes, cependant que dans le deuxième cas, c'est l'influence de certains facteurs: infection microbienne, infestation parasitaire, alimentation non équilibrée ou carencée, toxiques divers, qui, par les métabolites anormaux qui se produisent peuvent changer la conformation de la protéine enzymatique et, en conséquence, son édifice macromoléculaire. Dans cette dernière alternative on comprend que la modification de l'enzymogramme soit reversible. Si les influences anormales persistent longtemps, son aspect peut être toutefois définitivement changé. Dans notre cas, on remarque que, à la suite du métissage, il se produit une modification caractéristique des divers enzymogrammes, ce qui prouve, à la lumière des hypothèses faites plus haut, qu'a eu lieu une perturbation de l'arrangement macromoléculaire de l'enzyme. Malheureusement, nous ne pouvons décider sans ambiguïté pour l'une ou l'autre alternative, génétique ou chimique. On pourrait tout de même établir une corrélation entre les modifications de certains enzymogrammes et les propriétés morphophysiologiques des animaux. Ça serait très utile pour diriger judicieusement les opérations d'amélioration des animaux, indifféremment de la nature des modificiations moléculaires produites.

\section{CONCLUSION}

1. Le polymorphisme de la phosphatase alcaline du lait de vache varie avec la race.

2. Le plus grand nombre (4 fractions) des isozymes a été trouvé dans le lait provenant de vaches de race Holstein.

3. Par métissage de diverses races l'enzymogramme du lait est modifié d'une manière caractéristique. 
4. Indifféremment du mécanisme intime qui est la cause de cette modification elle pourrait servir à dépister et apprécier l'effet du métissage.

(Recu pour publication le 8 juillet 1968)

\section{S u m m a r y}

The chromatographic analysis (by gel filtration on Sephadex G-200) of the alkaline phosphatase from the cow milk has revealed its polymorphism.

The enzymograms are characteristic for the races studied. The greatest number of isozymes (4 fractions) was found for Holstein race (originated from Canada). The half-breeding of different races modifies distinctly the enzymograms. This could help to render evident and evaluate the result of the inbreeding.

\section{BIBLIOGRAPHIE}

[1] Buruiana (L. M.) et Badilitza (A.). - Etude sur les phosphatases du lait. 1 vol. 69 pages. 1938, Bucarest (en roumain).

[2] Buruiana (L. M.) et Litinsky (L.). - Nouvelles méthodes pour apprécier l'activité de l'amylase et de la phosphatase pour le contrôle de la pasteurisation basse. 1 vol. 57 pages. 1939, Bucarest (en roumain).

[3] Buruiana (L. M.) et Lerch (Marthe). - L'activité de la cathépsine de certaines fractions protéiques du muscle séparées électrophorétiquement. Travaux scientifiques de l'Institut Agronomique N. Balcesco, Bucarest. Série C. IX. 529, 1966.

[4] Buruiana (L. M.) et Lerch (M.). - La modification de l'enzymogramme de la cathépsine tissulaire après hybridation. Naturwiss. 1967. 54, 4, 92.

[5] Estborn (B.). - Separation of phosphatase isoenzymes by gel filtration. Zeitsch. $f$. Klin. Chemie. 1964. 2, 53.

[6] Folin (O.). - Laboratory Manual of Biological Chemistry. D. Appleton Century Comp. New York, Vth Edition. 339. 1934.

[7] Got (R.). - Presence of an acid phosphatase of the prostate type in human colostrum. Clin. Chim. Acta. 1964. 14, 6, 842.

[8] Hodson (A. W.), LATner (A. L.) and Raine (L.). - Isoenzymes of alkaline posphatase. Clin. Chim. Acta. 1962, 7. Nr. 1-3, 255.

[9] King (E.J.) and Armstrong (A.R.). - A convenient method for determining serum and bile phosphatase activity. Canad. Med. Assoc. 1934. J. 31, 376.

[10] Miller (G. L.). - Protein determination for large numbers of samples. Anal. Chem. 1959. 31 (53), 964.

[11] Nisselbaum (J. S.), Schlamowitz (M.), Bodansky (O.), - Immuonochemical studies of functionally similar enzymes. Ann. New-York Acad. Sci. 1961. 94, 3, 970.

[12] Perez (G.A.). - Distribution of enzymes in the protein fraction of human colostrum. Thèse Fac. Méd., Paris. 1967, Nr. 827. 